\title{
ON STOCHASTIC DIFFERENTIAL INCLUSIONS WITH CURRENT VELOCITIES
}

\author{
Yu.E. Gliklikh ${ }^{1}$, yeg@math.vsu.ru,
}

A.V. Makarova ${ }^{1}$, allagm@mail.ru.

${ }^{1}$ Voronezh State University, Voronezh, Russian Federation.

\begin{abstract}
Existence of solution theorems are obtained for stochastic differential inclusions given in terms of the so-called current velocities (symmetric mean derivatives, a direct analogs of ordinary velocity of deterministic systems) and quadratic mean derivatives (giving information on the diffusion coefficient) on the flat $n$-dimensional torus. Right-hand sides in both the current velocity part and the quadratic part are set-valued but satisfy the conditions, under which they have smooth selectors. Then we can reduce the inclusion to an equation with current velocities for which existence of solutions is known in the case of smooth right-hand side.

Keywords: mean derivatives; current velocities; differential inclusions.
\end{abstract}

\section{Introduction}

The notion of mean derivatives was introduced by Edward Nelson [8-10] for the needs of stochastic mechanics (a version of quantum mechanics). The equation of motion in this theory (called the Newton - Nelson equation) was the first example of equations in mean derivatives. Later it turned out that the equations in mean derivatives arose also in many other branches of science (mechanics, hydrodynamics, Navier - Stokes vortices, gauge fields, economics, etc.).

Nelson introduced forward and backward mean derivatives while only their half-sum, symmetric mean derivative called current velocity, is a direct analog of ordinary velocity for deterministic processes. In [2] another mean derivative called quadratic, is introduced. It gives information on the diffusion coefficient of the process and using Nelson's and quadratic mean derivatives together, one can in principle recover the process from its mean derivatives.

Since the current velocities are natural analogs of ordinary velocities of deterministic processes, investigation of equations and especially inclusions with current velocities is very much important for applications since there are a lot of models of various physical, economical etc. processes based on such equations and inclusions.

Differential inclusions with current velocities arise in natural problems of applications in the same way as ordinary differential inclusions originate from ordinary differential equations. Here we should point out two main cases. First, in equations with control and feedback, at every point $(t, x)$ of extended phase space one have to consider all values of the right-hand side for all possible values of controlling parameter. Thus, the right-had side becomes set-valued and the equation turns into inclusion.

The second case arises if the right-hand side of equation is "very bad" - neither continuous nor measurable - that often arise in description of motion in a very complicated medium. In this case the equation has no classical solution but there is a trick suggested probably by A.F. Filippov, that transforms the equation into inclusion with upper semicontinuous right hand side with convex images. Thus, the investigation of differential 
inclusions with mean derivatives is important for applications and it is a natural extension of the domain of applications for this theory.

In [2] an existence theorem for differential inclusions with current velocities having single-valued part for quadratic mean derivative, is obtained under some very strong conditions. In this paper we deal with more realistic situation. Both current velocity and quadratic parts of the right-hand side are set-valued. We use some criteria for existence of smooth selectors of set-valued mappings in order to reduce the inclusion to an equation with current velocities, for which existence of solutions is proved in [2] in the case of smooth right-hand side.

To avoid some technical difficulties we consider the inclusions on the flat $n$-dimensional torus $\mathcal{T}^{n}$. This means that the torus is considered as a quotient space of $\mathbb{R}^{n}$ relative to the integral lattice and that the Riemannian metric on $\mathcal{T}^{n}$ is inherited from the Euclidean metric in $\mathbb{R}^{n}$. Everywhere below we use the operations of addition and subtraction of points and integration in $\mathcal{T}^{n}$ as in $\mathbb{R}^{n}$ modulo factorization relative to the integral lattice. The construction and notation of stochastic integrals and stochastic differential equations on $\mathcal{T}^{n}$ are the same as in $\mathbb{R}^{n}$ because of the use of Euclidean metric.

The detailed exposition of preliminary notions and facts used in the paper, can be found in [7].

Everywhere in the paper we use Einstein's convention of summation relative to a shared upper and lower index (see, e.g., [7]).

\section{Preliminaries on Mean Derivatives}

Consider the $n$-dimensional flat torus $\mathcal{T}^{n}$. We shall deal with stochastic processes in $\mathcal{T}^{n}$ given on a certain probability space $(\Omega, \mathcal{F}, \mathrm{P}), t \in[0, T] \subset \mathbb{R}$.

Denote by $\mathcal{P}_{t}^{\xi}$ the sub- $\sigma$-algebra of $\mathcal{F}$ generated by preimages of Borel sets from $\mathcal{T}^{n}$ by all mappings $\xi(s): \Omega \rightarrow \mathbb{R}^{n}$ for $0 \leq s \leq t$; $\mathcal{P}_{t}^{\xi}$ is called the "past" for $\xi(t)$.

Denote by $\mathcal{N}_{t}^{\xi}$ the sub- $\sigma$-algebra of $\mathcal{F}$ generated by preimages of Borel sets from $\mathcal{T}^{n}$ by the mapping $\xi(t): \Omega \rightarrow \mathcal{T}^{n} ; \mathcal{N}_{t}^{\xi}$ is called the "present" for $\xi(t)$.

The $\sigma$-subalgebras $\mathcal{P}_{t}^{\xi}$ and $\mathcal{N}_{t}^{\xi}$ for all $t$ are supposed to be complete, i.e., containing all sets of probability zero. Obviously $\mathcal{N}_{t}^{\xi}$ is a sub- $\sigma$-algebra in $\mathcal{P}_{t}^{\xi}$.

For the sake of convenience we denote by $E_{t}^{\xi}$ the conditional expectation $E\left(\cdot \mid \mathcal{N}_{t}^{\xi}\right)$ with respect to $\mathcal{N}_{t}^{\xi}$ for $\xi(t)$.

As in [8-10], we introduce the following notions of forward and backward mean derivatives.

Definition 1. (i) The forward mean derivative $D \xi(t)$ of $\xi(t)$ at the time instant $t$ is an $L_{1}$ random element of the form

$$
D \xi(t)=\lim _{\triangle t \rightarrow+0} E_{t}^{\xi}\left(\frac{\xi(t+\triangle t)-\xi(t)}{\triangle t}\right)
$$

where the limit is supposed to exist in $L_{1}(\Omega, \mathcal{F}, \mathrm{P})$ and $\triangle t \rightarrow+0$ means that $\triangle t$ tends to 0 and $\triangle t>0$.

(ii) The backward mean derivative $D_{*} \xi(t)$ of $\xi(t)$ at $t$ is the $L_{1}$-random element

$$
D_{*} \xi(t)=\lim _{\Delta t \rightarrow+0} E_{t}^{\xi}\left(\frac{\xi(t)-\xi(t-\Delta t)}{\Delta t}\right)
$$


where (as well as in (i)) the limit is assumed to exist in $L^{1}(\Omega, \mathcal{F}, \mathrm{P})$ and $\Delta t \rightarrow+0$ means that $\Delta t \rightarrow 0$ and $\Delta t>0$.

As usual in the machinery of conditional expectation (see, e.g., [11]), there exist Borel measurable vector fields $a^{\xi}(t, m)$ and $a_{*}^{\xi}(t, m)$ such that $D \xi(t)=a^{\xi}(t, \xi(t))$ and $D_{*} \xi(t)=a_{*}^{\xi}(t, \xi(t))$, respectively.

Definition 2. The derivative $D_{S}=\frac{1}{2}\left(D+D_{*}\right)$ is called the symmetric mean derivative. The derivative $D_{A}=\frac{1}{2}\left(D-D_{*}\right)$ is called the antisymmetric mean derivative.

Consider the vectors $v^{\xi}(t, x)=\frac{1}{2}\left(a^{\xi}(t, x)+a_{*}^{\xi}(t, x)\right)$ and $u^{\xi}(t, x)=\frac{1}{2}\left(a^{\xi}(t, x)-a_{*}^{\xi}(t, x)\right)$.

Definition 3. $v^{\xi}(t)=v^{\xi}(t, \xi(t))=D_{S} \xi(t)$ is called the current velocity of the process $\xi(t)$; $u^{\xi}(t)=u^{\xi}(t, \xi(t))=D_{A} \xi(t)$ is called the osmotic velocity of the process $\xi(t)$.

The physical meaning of current velocity is a direct analog of the ordinary velocity of a deterministic process. The osmotic velocity measures how fast the randomness increases. This interpretation becomes clear from the following features of $v^{\xi}$ and $u^{\xi}$ (see [10]).

Consider an autonomous smooth field of non-degenerate linear operators $A(t, x): \mathbb{R} \times \mathbb{R}^{n} \rightarrow \mathbb{R}^{n}, t \in \mathbb{R}$ and $x \in \mathcal{T}^{n}$. Suppose that $\xi(t)$ is a diffusion type process whose diffusion integrand is $A(t, \xi(t))$. Then its diffusion coefficient $A(t, x) A^{*}(t, x)$ is a smooth field of symmetric positive definite $(2,0)$-tensors with matrices $\alpha(t, x)=\left(\alpha^{i j}(t, x)\right)$. Since all those matrices are non-degenerate, the field of inverse matrices $\left(\alpha_{i j}(t, x)\right)$ exists and is smooth and at any $(t, x)$ the matrix $\left(\alpha_{i j}\right)(t, x)$ is symmetric and positive definite. Thus it defines a new Riemannian metric (symmetric positive definite $(0,2)$-tensor field) $\alpha_{t}(\cdot, \cdot)=\alpha_{i j} d x^{i} \otimes d x^{j}$ on the level surfaces $t=$ const. Consider the Riemannian volume form of this Riemannian metric $\Lambda_{\alpha_{t}}=\sqrt{\operatorname{det}\left(\alpha_{i j}\right)} d x^{1} \wedge d x^{2} \wedge \cdots \wedge d x^{n}$ on those level surfaces.

Denote by $\rho^{\xi}(t, x)$ the probability density of $\xi(t)$ with respect to the volume form $d t \wedge \Lambda_{\alpha}=\sqrt{\operatorname{det}\left(\alpha_{i j}\right)} d t \wedge d x^{1} \wedge d x^{2} \wedge \cdots \wedge d x^{n}$ on $[0, T] \times \mathcal{T}^{n}$, i.e., for any continuous bounded function $f:[0, T] \times \mathcal{T}^{n} \rightarrow \mathbb{R}$ the relation

$$
\int_{0}^{T} E(f(t, \xi(t))) d t=\int_{0}^{T}\left(\int_{\Omega} f(t, \xi(t)) d \mathrm{P}\right) d t=\int_{0}^{T}\left(\int_{\mathbb{R}^{n}} f(t, x) \rho^{\xi}(t, x) \Lambda_{\alpha_{t}}\right) d t
$$

holds. Then under the assumption that $\rho^{\xi}(t, x)$ nowhere equals zero

$$
u^{\xi}(t, x)=\frac{1}{2} \frac{\frac{\partial}{\partial x^{j}}\left(\alpha^{i j}(t, x) \rho^{\xi}(t, x)\right)}{\rho^{\xi}(t, x)} \frac{\partial}{\partial x^{i}}
$$

where $\left(\alpha^{i j}\right)$ is the matrix of operator $A A^{*}$. Formula (4) is proved in [4].

For $v^{\xi}(t, x)$ and $\rho^{\xi}(t, x)$ the so called equation of continuity

$$
\frac{\partial \rho^{\xi}(t, x)}{\partial t}=-\operatorname{Div}_{t}\left(v^{\xi}(t, x) \rho^{\xi}(t, x)\right)
$$

holds, where $\operatorname{Div}_{t}$ denotes divergence with respect to the Riemannian metric $\alpha_{t}(\cdot, \cdot)$ on the level surface $t=$ const. Formula (5) is proved in [2]. 
Following [2] we introduce the differential operator $D_{2}$ that differentiates an $L_{1}$ random process $\xi(t), t \in[0, T]$ according to the rule

$$
D_{2} \xi(t)=\lim _{\triangle t \rightarrow+0} E_{t}^{\xi}\left(\frac{(\xi(t+\triangle t)-\xi(t))(\xi(t+\Delta t)-\xi(t))^{*}}{\triangle t}\right),
$$

where $(\xi(t+\triangle t)-\xi(t))$ is considered as a column vector (vector in $\left.\mathbb{R}^{n}\right),(\xi(t+\triangle t)-\xi(t))^{*}$ is a row vector (transposed, or conjugate vector) and the limit is supposed to exists in $L_{1}(\Omega, \mathcal{F}, \mathrm{P})$. We emphasize that the matrix product of a column on the left and a row on the right is a matrix with rank 1 but after passing to limit and taking conditional expectation $D_{2} \xi(t)$ becomes a symmetric semi-positive definite matrix function on $[0, T] \times \mathbb{R}^{n}$ (in many cases positive definite). We call $D_{2}$ the quadratic mean derivative. It takes values in the set of $(2,0)$-tensors having symmetric positive semi-definite matrices.

As mentioned above, the current velocity is analogous to ordinary velocity for a nonrandom process. Thus, from the physical point of view, it is an important problem to study equations and inclusions with current velocities.

Let $v(t, m)$ be a vector field and $\alpha(t, m)$ be a symmetric positive semi-definite $(2,0)$ tensor field on $\mathcal{T}^{n}$. The system

$$
\left\{\begin{aligned}
D_{S} \xi(t) & =v(t, \xi(t)) \\
D_{2} \xi(t) & =\alpha(t, \xi(t))
\end{aligned}\right.
$$

is called the first order differential equation with current velocities.

Note that equation (7) on the flat torus $\mathcal{T}^{n}$ can be considered as an equation on $\mathbb{R}^{n}$ periodic in space variables.

Definition 4. We say that (7) on $\mathcal{T}^{n}$ has a solution on $[0, T]$ with initial condition $\xi(0)=\xi_{0}$ if there exists a probability space $(\Omega, \mathcal{F}, \mathrm{P})$ and a process $\xi(t)$ given on $(\Omega, \mathcal{F}, \mathrm{P})$ and taking values in $\mathcal{T}^{n}$ such that $\xi(0)=\xi_{0}$ and for almost all $t \in[0, T]$ equation (7) is satisfied $\mathrm{P}-$ a.s. by $\xi(t)$.

Theorem 1. Let $v:[0, T] \times \mathcal{T}^{n} \rightarrow \mathbb{R}^{n}$ and $\alpha:[0, T] \times \mathcal{T}^{n} \rightarrow \mathrm{S}_{+}(n)$ be smooth (so $\alpha$ determines the Riemannian metric $\alpha_{t}(\cdot, \cdot)$ on $\mathcal{T}^{n}$, introduced above). Let $\xi_{0}$ be a random element with values in $\mathcal{T}^{n}$ whose probability density $\rho_{0}$ with respect to the volume form $\Lambda_{\alpha}$ of $\alpha(\cdot, \cdot)$ on $\mathcal{T}^{n}$ (see above) is smooth and nowhere equal to zero. Then for the initial condition $\xi(0)=\xi_{0}$ equation (7) has a solution that is well-defined on the entire interval $t \in[0, T]$.

Theorem 1 is a simple corollary to [2, Theorem 3]. Here we use the fact that on the compact manifold $\mathcal{T}^{n}$ the right-hand sides of (7) are uniformly bounded and so the hypothesis of [2, Theorem 4.1] is fulfilled.

Introduce $p_{0}=\log \rho_{0}$ and consider $p(t, m)=\log \rho^{\xi}(t, m)$ where $\rho^{\xi}(t, m)$ is the density (3) corresponding to the solution $\xi(t)$ of $(7)$. It is shown in [2, Theorem 3] that $p(t, m)$ is well-posed and takes the form

$$
p(t, m)=p_{0}\left(g_{-t}(m)\right)-\int_{0}^{t}\left(\operatorname{Div}_{s} v\right)\left(s, g_{s}\left(g_{-t}(m)\right) d s,\right.
$$

where $\operatorname{Div}_{t}$ is the divergence with respect to $\alpha_{t}(\cdot, \cdot)$ on the level surface $t=$ const and $g_{t}$ is the flow of smooth vector field $v(t, m)$. 


\section{Inclusions with Current Velocities}

Let $\mathbf{v}(t, m)$ be a set-valued vector field and $\boldsymbol{\alpha}(t, m)$ a set-valued symmetric positive semi-definite $(2,0)$-tensor field on $\mathcal{T}^{n}$. The system of the form

$$
\left\{\begin{array}{l}
D_{S} \xi(t) \in \mathbf{v}(t, \xi(t)) \\
D_{2} \xi(t) \in \boldsymbol{\alpha}(t, \xi(t))
\end{array}\right.
$$

is called a first order differential inclusion with current velocities. The notion of solution of (9) is quite analogous to that from Definition 4.

Theorem 2. Let $\mathbf{v}(t, m)$ be a set-valued uniformly bounded vector field on $\mathcal{T}^{n}$ that has a smooth selector. Let also $\boldsymbol{\alpha}(t, m)$ be a $(2,0)$-tensor field having a smooth selector as well and taking values in $S_{+}(n)$. In addition, let $\xi_{0}$ be a random element with values in $\mathcal{T}^{n}$ whose density $\rho_{0}$ is smooth and nowhere equal to zero. Then for initial condition $\xi(0)=\xi_{0}$ inclusion (9) has a solution that exists on the entire interval $t \in[0, T]$.

Proof.

Denote the smooth selector of $\boldsymbol{\alpha}(m)$ by $\alpha(t, m)$ and of $\mathbf{v}(t, m)$ by $v(t, m)$. It is evident that the density of $\xi_{0}$ with respect to the volume form of Riemannian metric constructed from $\alpha(t, m)$ as above, is nowhere equal to zero. Then by Theorem 1 equation

$$
\left\{\begin{array}{l}
D_{S} \xi(t)=v(t, \xi(t)) \\
D_{2} \xi(t)=\alpha(t, \xi(t))
\end{array}\right.
$$

has a solution well-posed on $t \in[0, T]$ and it is a solution of (9) we are looking for.

Now we can apply the criteria for existence of smooth selectors for set-valued mappings to obtain new existence theorems for inclusion (9).

For a solid closed convex set $A$ and vector $V$ in $\mathbb{R}^{n}$ let us introduce the so-called support function $\Psi(A, V)=\sup _{y \in A}(y, V)$ where $(\cdot, \cdot)$ is inner product. Consider the set-valued vector field $\mathbf{v}(t, m)$ on $\mathcal{T}^{n}$ whose values are solid, convex and closed. Since the tangent bundle of torus is trivial, we can deal with the constant vector field $V$ on the torus. Denote by $\Psi(t, m, V)$ that support function of $\Psi(\mathbf{v}(t, m), V)$. Let also the (2,0)-tensor field $\boldsymbol{\alpha}(t, m)$ on $\mathcal{T}^{n}$ has values solid, convex and closed. Since $\boldsymbol{\alpha}(t, m)$ takes values in $S_{+}(n) \subset L\left(\mathbb{R}^{n}, \mathbb{R}^{n}\right)$ that is a linear space, we can construct the support function $\Psi_{1}\left(t, m, V_{1}\right)$ for $\boldsymbol{\alpha}(t, m)$ by complete analogy with the above scheme where $V_{1}$ is a constant $(2,0)$-tensor field on $\mathcal{T}^{n}$.

Assumption 1. For every $V$ and $V_{1}$ functions $\Psi(t, m, V)$ and $\Psi_{1}\left(t, m, V_{1}\right)$ are smooth.

Theorem 3. Let $\mathbf{v}(t, m)$ be a continuous uniformly bounded set-valued vector field on $\mathcal{T}^{n}$ having solid convex and closed values and let also $\boldsymbol{\alpha}(t, m)$ be a continuous uniformly bounded set-valued $(2,0)$-tensor field on $\mathcal{T}^{n}$ such that its values are solid convex and closed. Suppose that Assumption 1 is fulfilled. In addition, let a random element $\xi_{0}$ with values in $\mathcal{T}^{n}$ has density that equals zero nowhere. Then for initial condition $\xi(0)=\xi_{0}$ inclusion (9) has a solution that is well-posed on the entire interval $t \in[0, T]$. 
Proof.

In [1] it is shown that under Assumption 1 the continuous set-valued uniformly bounded vector field with closed convex solid values has a smooth selector. Thus, the statement of Theorem follows from Theorem 2.

Now let us consider the case where the values of $\mathbf{v}(t, m)$ and $\boldsymbol{\alpha}(t, m)$ are not solid. Instead we suppose that every image of $\mathbf{v}(t, m)$ (of $\boldsymbol{\alpha}(t, m)$ ) belongs to a subspace in $T_{m} \mathcal{T}^{n}$ (a subspace in the space of $(2,0)$ tensors at $m$, respectively) and those subspaces have constant dimension $k<n$ ( $k_{1}<n$, respectively) independent of $m \in \mathcal{T}^{n}$. If we associate with every $m \in \mathcal{T}^{n}$ the above-mentioned subspace at $m$, we obtain a mapping $\Phi$ form $\mathcal{T}^{n}$ to the manifold of affine subspaces with dimension $k$ in tangent spaces to $T_{m} \mathcal{T}^{n}$ (mapping $\Phi_{1}$ to the manifold of affine subspaces in the spaces of $(2,0)$-tensors, respectively).

Assumption 2. Mappings $\Phi$ and $\Phi_{1}$ are smooth.

Theorem 4. Let $\mathbf{v}(t, m)(\boldsymbol{\alpha}(t, m))$ be a continuous uniformly bounded set-valued vector field $\left((2,0)\right.$-tensor field with values in $\left.S_{+}(n)\right)$ on $\mathcal{T}^{n}$ such that every image $\mathbf{v}(t, m)$ belongs to a subspace in $T_{m} \mathcal{T}^{n}$ (every image $\boldsymbol{\alpha}(t, m)$ belongs to a subspace in the space of $(2,0)$ tensors, respectively) and those subspaces have constant dimension $k<n\left(k_{1}<n\right.$, respectively) independent of $m \in \mathcal{T}^{n}$. Let also Assumption 2 be fulfilled. Then for initial condition $\xi(0)=\xi_{0}$ where the density of $\xi_{0}$ is smooth and nowhere equal to zero, inclusion (9) has a solution that is well-posed on the entire interval $t \in[0, T]$.

Proof.

In [5] it is shown that in the case under Assumption 2, the continuous uniformly bounded vector field with closed convex images has a smooth selector. Thus, the statement of Theorem follows from Theorem 2.

In the case where the right-hand side of (9) is not continuous but upper or lower semicontinuous, we have to construct smooth $\varepsilon$-approximations in the former case and smooth selectors in the latter case (see [3]). Taking those approximations, we construct equations with current velocities of (7) type and after that we have to prove that the solutions of the sequence of equations converge to the solution of (9). The following Theorem can be a useful tool in this case.

Consider a sequence of equations of (7) type on $\mathcal{T}^{n}$ whose right-hand sides are smooth and uniformly bounded for all $k$ by the same constant. For $k$-th equation denote by $\mu_{k}$ the measure on the space of continuous paths $\left(C^{0}\left([0, T], \mathcal{T}^{n}\right), \mathcal{C}\right)$ (where $\mathcal{C}$ is the $\sigma$-algebra generated by cylinder sets) corresponding to the solution $\xi_{k}(t)$ of the $k$-th equation.

Theorem 5. The set of measures $\mu_{k}$ on $\left(C^{0}\left([0, T], \mathcal{T}^{n}\right), \mathcal{C}\right)$ is weakly compact.

Proof.

First of all, note that since all processes $\xi_{k}(t)$ take values in the compact torus, all expectations $E \xi_{k}(t)$ are uniformly bounded.

In [2], on the basis of Hauss decomposition (see [12]), every matrix $\alpha \in S_{+}(n)$ is represented in the form $\alpha=\zeta \delta \zeta^{*}$ where $\zeta$ is a lower-triangle matrix with units on the 
diagonal, $\zeta^{*}$ is its transposed matrix, i.e., an upper-triangle matrix with units on the diagonal, and $\delta$ is a diagonal matrix whose angular minors (note that they all a positive) coincide with those of $\alpha$. Denote the diagonal elements of $\delta$ by $\delta_{1}, \ldots \delta_{n}$. Then the matrix $A=\zeta \sqrt{\delta}$ where $\sqrt{\delta}$ is the diagonal matrix with $\sqrt{\delta_{1}}, \ldots, \sqrt{\delta_{n}}$ on the diagonal, is such that $\alpha=A A^{*}$. If we deal with a smooth field $\alpha_{k}(t, m), t \in \mathbb{R}$ and $m \in \mathcal{T}^{n}$, of the abovementioned approximations, the corresponding matrices $A_{k}(t, m)$ are also smooth. Since $\alpha_{k}(t, m)$ are uniformly bounded by the same constant for all $k$, all $A_{k}(t, m)$ have the same property.

Specify two real numbers $0 \leq s<t \leq T$ with small difference $t-s$. Then for any $k$ the increment of $\xi_{k}$ on $[s, t]$ is approximated by $v_{k}\left(\frac{s+t}{2}\right)(t-s)+A_{k}(s)(w(t)-w(s))$. Consider $E\left(\left(v_{k}\left(\frac{s+t}{2}\right)(t-s)+A_{k}(s)(w(t)-w(s))\right)\left(v_{k}\left(\frac{s+t}{2}\right)(t-s)+A_{k}(s)(w(t)-w(s))\right)^{*}\right)$. Since $v_{k}$ and $A_{k}$ are uniformly bounded for all $k$ by a unique constant, one can easily see that among the items in the obtained expression only $\alpha_{k}(t-s)$ is infinitesimal of the same order as $t-s$ while the other items are infinitesimals of a higher order. Hence there exists a constant $h_{1}$ such that if the difference $t-s$ is small enough, the above-mentioned expression is not greater than $h_{1}(t-s)$. By integration one can show that there exists a constant $h>0$ depending on $T$ and on the constant that bounds the norms of $v_{k}$ and $\alpha_{k}$, such that for any $0 \leq t_{1}<t_{2} \leq T$ and every $k$ the inequality $E\left(\xi_{k}\left(t_{2}\right)-\xi_{k}\left(t_{1}\right)\right)^{4}<h\left(t_{2}-t_{1}\right)^{2}$ holds. Now the statement of Theorem follows from [6, Theorem $2 \S 4$ of Chapter VI].

The research is supported in part by RFBR Grant No. 15-01-00620.

\section{References}

1. Aseev S.M. [Existence of Differentiable Single-Valued Branch for the Set-Valued Mapping]. Nekotorye voprosy prikladnoy matematiki i programmnogo obespecheniya EVM [Some Questions of Applied Mathematics and Computer Software of PC]. Moscow, Moscow University Publ., 1982, pp. 36-39. (in Russian)

2. Azarina S.V., Gliklikh Yu.E. Differential Inclusions with Mean Derivatives. Dynamic Systems and Applications, 2007, vol. 16, no. 1, pp. 49-71.

3. Borisovich Yu.G., Gelman B.D., Myshkis A.D., Obukhovskii V.V. Vvedenie v teoriyu mnogoznachnykh otobrazheniy $i$ differentsial'nykh vklyucheniy [Introduction to the Theory of Multi-Valued Mappings and Differential Inclusions]. Moscow, Komkniga Publ., 2005.

4. Cresson J., Darses S. Stochastic Embedding of Dynamical Systems. Journal of Mathematical Physics, 2007, vol. 48, pp. 072703-1-072303-54.

5. Farber M.Sh. [On Smooth Selectors of the Intersection of Set-Valued Mappings]. Izvestiya Akademii Nauk Azerbaijanskoy SSR [Proceedings of the Academy of Sciences of Azerbaijan SSR], 1979, no. 6, pp. 23-28. (in Russian)

6. Gikhman I.I., Skorokhod A.V. The Theory of Stochastic Processes I. New York, Springer-Verlag, 1974.

7. Gliklikh Yu.E. Global and Stochastic Analysis with Applications to Mathematical Physics. London, Springer-Verlag, 2011. DOI: 10.1007/978-0-85729-163-9 
8. Nelson E. Derivation of the Schrödinger Equation from Newtonian Mechanics. Physical Reviews, 1966, vol. 150, issue 4, pp. 1079-1085.

9. Nelson E. Dynamical Theory of Brownian Motion. Princeton, Princeton University Press, 1967.

10. Nelson E. Quantum Fluctuations. Princeton, Princeton University Press, 1985.

11. Parthasarathy K.R. Introduction to Probability and Measure. New York, SpringerVerlag, 1978.

12. Zhelobenko D.P. Compact Lie Groups and Their Representations. Amer. Math. Soc., Providence, RI, 1973.

Yuri E. Gliklikh, doctor of physical and mathematical sciences, full professor, department of algebra and topological analysis methods, Voronezh State University, Voronezh, Russian Federation,yeg@math.vsu.ru.

Alla V. Makarova, engineer, mathematics faculty, Voronezh State University, Voronezh, Russian Federation, allagm@mail.ru.

Received August 20, 2015

УДК 519.216.2

DOI: $10.14529 /$ jcem150303

\title{
О СТОХАСТИЧЕСКИХ ДИФФЕРЕНЦИАЛЬНЫХ ВКЛЮЧЕНИЯХ С ТЕКУЩИМИ СКОРОСТЯМИ
}

\author{
Ю.Е. Гликлих, А.В. Макарова
}

\begin{abstract}
Получены теоремы существования решений стохастических дифференциальных включений, заданных в терминах так называемых текущих скоростей (симметрических производных в среднем, прямых аналогов обычных скоростей детерминированных систем) и квадратичных производных в среднем (дающих информацию о коэффициенте диффузии) на плоском $n$-мерном торе. Правые части и для текущей скорости, и для для квадратичной производной многозначны и удовлетворяют условиям, при которых они имеют гладкие селекторы. Затем включение сводится к уравнению с текущими скоростями, для которого теорема существования решения известна в случае гладкой правой части.

Ключевые слова: производные в среднем; текущие скорости; дифференциальные включения.
\end{abstract}

\section{Литература}

1. Асеев, С.М. Существование дифференцируемой однозначной ветви для многозначных отображений / C.M. Асеев // Некоторые вопросы прикладной математики и программного обеспечения ЭВМ. - 1982. - С. 36-39.

2. Azarina, S.V. Differential inclusions with mean derivatives / S.V. Azarina, Yu.E. Gliklikh // Dynamic systems and applications. - 2007. - V. 16. - P. 49-72. 
3. Борисович, Ю.Г. Введение в теорию многозначных отображений и дифференциальных включений / Ю.Г. Борисович, Б.Д. Гельман, А.Д. Мышкис, В.В. Обуховский. - М.: Комкнига, 2005. - 213 с.

4. Cresson, J. Stochastic Embedding of Dynamical Systems / J. Cresson, S. Darses // Journal of Mathematical Physics.- 2007. - V. 48. - P. 072703-1-072303-54.

5. Фарбер, М.Ш. О гладких селекторах пересечений многозначных отображений / М.Ш. Фарбер // Известия Академии наук Азербайджанской ССР. - 1979. - № 6. C. $23-28$.

6. Гихман, И.И. Теория случайных процессов. Т. 1 / И.И. Гихман, А.В. Скороход. - М.: Физматлит, 1971. - 664 с.

7. Гликлих, Ю.Е. Глобальный и стохастический анализ в задачах математической физики / Ю.Е. Гликлих. - М.: Комкнига, 2005. - 416 с.

8. Nelson, E. Derivation of the Schrödinger Equation from Newtonian Mechanics / E. Nelson // Physical Reviews. - 1966. - V. 150. - P. 1079-1085.

9. Nelson, E. Dynamical Theory of Brownian Motion / E. Nelson. - Princeton: Princeton University Press, 1967. - 142 p.

10. Nelson, E. Quantum Fluctuations / E. Nelson. - Princeton: Princeton University Press, 1985. - $147 \mathrm{p}$.

11. Партасарати, К.Р. Введение в теорию вероятностей и теорию меры / К.Р. Партасарати. - М.: Мир, 1988. - 344 с.

12. Желобенко, Д.П. Компактные группы Ли и их представления / Д.П. Желобенко. - М.: Физматлит, 1970. - 674 с.

Гликлих Юрий Евгенъевич, доктор физико-математических наук, профессор, кафедра алгебры и топологических методов анализа, Воронежский государственный университет (2. Воронеж, Российская Федерация), уеg@таth.vsu.ru.

Макарова Алла Викторовна, инженер, математический факультет, Воронежский государственный университет (2. Воронеж, Российская Федерачия), allagm@mail.ru.

Поступила в редакцию 20 августа 2015 г. 